\title{
Ciclo Biológico em Laboratório de Rhodnius brethesi Matta, 1919 (Hemiptera, Reduviidae, Triatominae), Potencial Vetor Silvestre da Doença de Chagas na Amazônia
}

\author{
Dayse da Silva Rocha+ , Carolina Magalhães dos Santos, Vanda Cunha, José Jurberg, \\ Cleber Galvão
}

Laboratório Nacional e Internacional de Referência em Taxonomia de Triatomíneos, Departamento de Entomologia, Instituto Oswaldo Cruz-Fiocruz Av. Brasil 4365, 21045-900 Rio de Janeiro, RJ, Brasil

\begin{abstract}
Life cycle of Rhodnius brethesi Matta, 1919 (Hemiptera, Reduviidae, Triatominae), a potential vector of Chagas disease in the Amazon region - R. brethesi is a sylvatic species from the Amazon region; it has been incriminated as responsible for the transmission of Chagas disease in collectors of piaçaba in this region. The aim of present study was to investigate the efficiency of these insects as potential vectors of Trypanosoma cruzi. Aspects related with feeding and defecation patterns, life time, and mortality had been observed in each instar of $\mathrm{R}$. brethesi. We use 5th instar nymphs to get adults virgins, after the moulting 3 groups with 6 females and 2 males each were created to obtain eggs. After hatching, 1st instar nymphs had been weighed and kept in bottles until the next moult. Insects were fed once a week in mice. Results showed that the average period of incubation was 17 days, the number of blood meal was increasing from the 1st to the 5th instar nymph with 7 (average) to become adult, a significative numbers of the defecations occurring immediately after the bloodmeals. The total percentual of mortality was $16 \%$. This results suggests that this species presents a good exploitation of blood meals and a brief nymphal development in laboratory conditions reflecting its behavior in sylvatic environments.
\end{abstract}

Key words: Chagas disease - Brazilian Amazon - Triatominae - life cycle - Rhodnius brethesi

Nos últimos anos a possibilidade de emergência e dispersão da doença de Chagas humana no território da Amazônia brasileira tem chamado atenção de alguns autores, pois, nesta região, são encontradas algumas espécies de triatomíneos em focos silvestres, bem como uma considerável circulação do Trypanosoma cruzi entre os vetores e reservatórios (Valente \& Valente 1993, Coura et al. 1999, 2002, Dias et al. 2002).

Alguns fatores são considerados pelos pesquisadores que trabalham nesta região como importantes desencadeadores de uma alteração no quadro atual da doença de Chagas humana, que presentemente é considerada uma enzootia silvestre na região amazônica. Entre esses fatores, podemos citar as migrações humanas e o crescente desmatamento da região. Atualmente, a doença apresenta um quadro difuso com casos esporádicos e ainda poucos relatos de triatomíneos encontrados em domicílios (Coura et al. 1999, Dias 2002).

Até o momento foram encontradas 18 espécies de triatomíneos na região da Amazônia brasileira: Alberprosenia malheroi, Belminus laportei, Cavernicola lenti, C. pilosa, Eratyrus mucronatus, Microtriatoma trinidadensis, Panstrongylus geniculatus, P. lignarius, P. megistus, P. rufotuberculatus, Rhodnius amazonicus, $R$. brethesi, $R$. paraensis, $R$. pictipes, $R$. prolixus, $R$.

Auxílio: CNPq, Faperj, convênio Funasa/Fiocruz/Fiotec, Chagas Disease Intervention Activities (CDIA)

+Author de contato. Fax:+55-21-2598.4321. E-mail: dsrocha@ioc.fiocruz.br

Recebido em 6 de abril de 2004

Aceito em 25 de agosto 2004 robustus, Triatoma maculata e T. rubrofasciata (Galvão et al. 2003). Dentre as espécies relatadas, $R$. brethesi merece uma atenção especial pela possibilidade de estar envolvido em um foco de transmissão silvestre da doença de Chagas em coletores de piaçaba no Alto Rio Negro, Amazonas (Dias et al. 2002). Estudos realizados por Coura et al. (1999) demonstraram que indivíduos soropositivos para anticorpos de T. cruzi, relataram a presença de triatomíneos em seus locais de trabalho (piaçabais), mencionando também a ocorrência de picadas nas cabanas utilizadas como abrigo nestas áreas; entretanto, não houve nenhum relato sobre o encontro de triatomíneos por estes trabalhadores em suas residências na cidade de Barcelos, Amazonas.

$R$. brethesi encontra-se distribuído no Norte e Nordeste do Brasil (estados do Amazonas, Maranhão e Pará), Colômbia e Venezuela. Sua distribuição altilatitudinal varia de 0 a 500 m.s.n.m. e de $10^{\circ} \mathrm{N}$ a $10^{\circ} \mathrm{S}$ (Rebelo et al. 1998, Carcavallo et al. 1999, Galvão et al. 2003). Esta espécie silvestre é comumente encontrada em palmeiras (Leopoldina piassaba), sendo conhecida popularmente como "piolho da piaçaba" entre a população desta região. Em 1971, D’Alessandro et al. incriminaram-na como vetor natural do $T$. rangeli na Colômbia.

O conhecimento de aspectos da biologia de espécies silvestres, é de grande importância para a avaliação da eficiência destes insetos como vetores do T. cruzi. O ciclo de vida dos triatomíneos varia de acordo com a espécie e condições ambientais onde vivem e é fortemente influenciado pela disponibilidade de fontes sangüineas adequadas (Schofield 1985). Da mesma forma, outros aspectos da biologia destes insetos são muito específicos, e a generalização dos resultados obtidos em algumas 
espécies para outras ainda não estudadas pode levar a conclusões equivocadas. Uma vez que os triatomíneos são ectoparasitas temporários, uma das características biológicas que apresenta maior impacto na epidemiologia da doença de Chagas envolve o momento do repasto sangüíneo e da defecação, pois só neste momento ocorre um íntimo contato com seus hospedeiros podendo assim adquirir ou transmitir o T. cruzi. Com o objetivo de avaliar a competência vetorial desta espécie foram avaliados os seguintes aspectos biológicos: período de incubação dos ovos, período de duração de cada estádio, número de repastos realizados em cada fase de desenvolvimento, percentuais de mortalidade em cada estádio, quantidade de sangue ingerido em cada estádio ninfal, percentual de defecações realizadas em até 10 min após o repasto e o período de duração do ciclo de ovo a adulto.

\section{MATERIAIS E MÉTODOS}

Foram separadas ninfas de $5^{\circ}$ estádio de $R$. brethesi para obtenção de adultos virgens. Após a muda foram formados 3 grupos compostos de 6 fêmeas e 2 machos, para obtenção de ovos. Os ovos foram individualizados, numerados e observados diariamente até a eclosão. Após a eclosão as ninfas de $1 \underline{0}$ estádio foram pesadas e mantidas em frascos de centrífuga de $50 \mathrm{ml}$ até a muda imaginal. Só foram retiradas deste frasco no momento da alimentação.

A primeira alimentação foi realizada 5 dias após a eclosão; a fonte sangüínea foi oferecida diariamente até a ocorrência do primeiro repasto, passando posteriormente a ser oferecida semanalmente. Os camundongos (Mus musculus) utilizados foram anestesiados segundo o protocolo 0014-00, aprovado pela Ceua-Fiocruz. Durante a alimentação os insetos foram colocados em um frasco de vidro de $13 \mathrm{~cm}$ de altura e $6 \mathrm{~cm}$ de diâmetro onde permaneciam por um período de $2 \mathrm{~h}$, quando eram pesados. Durante a alimentação também se observava a ocorrência de defecações que se estendiam por um período de até 10 min após o término do repasto. O experimento foi realizado em temperatura ambiente, sendo registradas durante o período de observações as temperaturas mínima de $19^{\circ} \mathrm{C}$, máxima $35^{\circ} \mathrm{C}$ e média $27^{\circ} \mathrm{C}$. Todas as observações foram feitas diariamente sendo registradas as ecdises e mortes. A fase experimental teve duração de 18 meses.

\section{RESULTADOS}

Período de incubação dos ovos e de desenvolvimento ninfal - O período médio de incubação dos ovos foi de 17 dias, com o mínimo de 7 e o máximo de 21 dias. Com relação ao desenvolvimento ninfal as médias obtidas não estão distribuídas de forma crescente. O menor período médio registrado foi observado no $2^{\mathrm{o}}$ estádio com 16 dias, seguido do $1^{\mathrm{O}} \operatorname{com} 18$, do $4^{\circ} \mathrm{o} \operatorname{com} 20$, do $3^{\mathrm{o}} \operatorname{com} 21$ e do $5^{\mathrm{o}}$ estádio com 38 dias. O período de desenvolvimento dos insetos do $1^{\mathrm{O}}$ estádio até a muda imaginal ocorreu em menos de quatro meses (115,3 dias), o que pode indicar que em condições favoráveis possam ocorrem até 3 gerações por ano (Tabela II).

Número de repastos - Os insetos necessitaram em média de 7 repastos para atingirem a fase adulta; do $1^{0}$ ao $5^{\circ}$ estádios os insetos realizaram em média menos de 2 repastos para atingirem o estádio seguinte, o que de- monstra o grande aproveitamento do sangue ingerido (Tabela I).

Quantidade de sangue ingerido - A quantidade de sangue ingerida foi crescente do $1^{0}$ ao $5^{\underline{0}}$ estádio, decaindo apenas na fase adulta, entretanto, o mesmo não foi observado para o aumento de peso corporal, onde observou-se o maior aumento das ninfas de $1 \underline{\underline{0}}, 2 \underline{\underline{0}}$ e $3 \underline{\underline{o}}$ estádios com aumento de 5,1 x; 5 x e 5,5 x respectivamente em relação às ninfas de 50 estádio com aumento corporal de 2,7 x (Tabela III).

Padrões de defecação - Na Tabela III estão relacionados os percentuais de defecações observados durante todo o ciclo em um período de até 10 min após o término da alimentação. Os percentuais observados foram variáveis; não houve uma correlação entre a quantidade de sangue ingerida e a ocorrência de defecações. O maior percentual de defecações observadas em até $10 \mathrm{~min}(82,8 \%)$ ocorreu no estádio adulto, sendo este estádio o que teve menor aumento de peso corporal. O menor percentual foi registrado no 10 estádio $(30,8 \%)$,

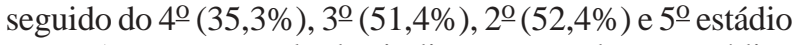
com $65 \%$. Estes resultados indicam que todos os estádios têm uma boa capacidade de transmissão em caso de alimentação em um hospedeiro infectado pelo T. cruzi.

Percentual de mortalidade - Das 54 ninfas que iniciaram o experimento, 13 atingiram a fase adulta (6 machos e 7 fêmeas). $\mathrm{O}$ maior percentual de mortalidade foi registrado no primeiro estádio $(10,8 \%)$ e o menor foi observado no $3 \underline{\mathrm{o}}$ estádio $(0,8 \%)$. Nos demais estádios de desenvolvimento ninfal os percentuais oscilaram entre 2,38 e $1,14 \%$ perfazendo um total de $16,3 \%$ de mortalidade durante todo o período (Fig. 1)

\section{TABELA I}

Número de repastos em cada fase de desenvolvimento de Rhodnius brethesi Matta, 1911

\begin{tabular}{lcccccc}
\hline Estádio & $\mathrm{N}$ & Min. & Max. & $\mathrm{X}$ & $\mathrm{S}$ & $\mathrm{S}^{2}$ \\
\hline I & 54 & 1 & 4 & 1,57 & 0,61 & 0,78 \\
II & 34 & 1 & 3 & 1,24 & 0,28 & 0,53 \\
III & 27 & 1 & 3 & 1,26 & 0,26 & 0,50 \\
IV & 24 & 1 & 3 & 1,32 & 0,29 & 0,53 \\
V & 19 & 1 & 4 & 1,73 & 0,67 & 0,81 \\
Adultos & 13 & 1 & 27 & 7,52 & 41,50 & 6,41 \\
\hline
\end{tabular}

N: número de insetos; X: média; S: desvio padrão; $S^{2}$ : variância

TABELA II

Período de desenvolvimento (em dias) de cada estádio de Rhodnius brethesi Matta, 1911

\begin{tabular}{lcccc}
\hline Estádio & $\mathrm{N}$ & Min. & Max. & $\mathrm{X} \pm \mathrm{S}^{2}$ \\
\hline Ovo- I & 54 & 7 & 21 & $17,04 \pm 2,05$ \\
I - II & 34 & 5 & 37 & $18,54 \pm 5,78$ \\
II - III & 27 & 2 & 29 & $16,03 \pm 5,69$ \\
III - IV & 24 & 8 & 30 & $21,26 \pm 5,12$ \\
IV - V & 19 & 13 & 38 & $20,92 \pm 6,01$ \\
V - Adultos & 13 & 18 & 66 & $38,61 \pm 11,23$ \\
\hline
\end{tabular}

N: número de insetos; I: $1^{\underline{o}}$ estádio; II: 2 o estádio; III: 3o estádio; IV: 4ํ estádio; V: 5o estádio 
TABELA III

Quantidade de sangue ingerido (mg), aumento de peso corporal e percentual de defecações realizadas em até 10 min após o repasto em Rhodnius brethesi Matta, 1911

\begin{tabular}{llccccc}
\hline Estádio & $\mathrm{N}$ & Min. & Max. & $\mathrm{X} \pm \mathrm{S}^{2}$ & APC & $\%$ defecação \\
\hline I & 54 & 0,1 & 5,7 & $1,9 \pm 1,4$ & $5,1 \mathrm{x}$ & 30,85 \\
II & 34 & 0,1 & 16,0 & $6,9 \pm 5,5$ & $5,0 \mathrm{x}$ & 52,38 \\
III & 27 & 0,3 & 59,1 & $30,2 \pm 20,6$ & $5,5 \mathrm{x}$ & 51,43 \\
IV & 24 & 0,6 & 184,4 & $76,4 \pm 56,9$ & $4,5 \mathrm{x}$ & 35,30 \\
V & 19 & 0,1 & 421,9 & $128,6 \pm 128,8$ & $2,7 \mathrm{x}$ & 65,00 \\
Adultos & 13 & 0,9 & 274,0 & $55,2 \pm 43,7$ & $1,4 \mathrm{x}$ & 82,83 \\
\hline
\end{tabular}

$\mathrm{N}$ : número de insetos; APC: aumento de peso corporal

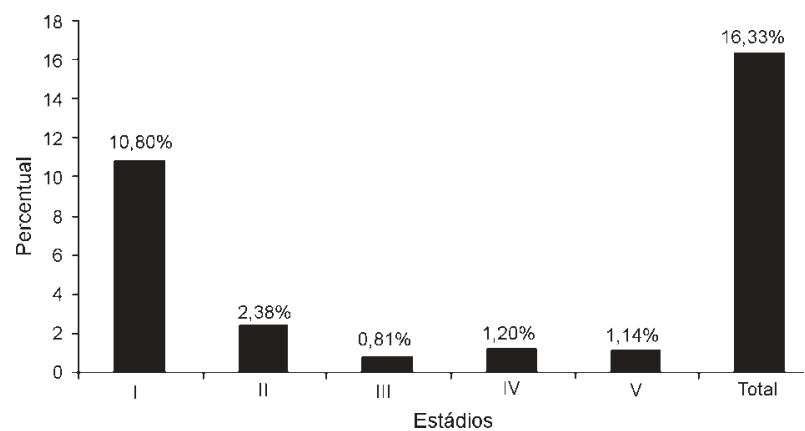

Percentual de mortalidade em cada estádio de desenvolvimento de Rhodnius brethesi Matta, 1911

\section{DISCUSS̃̃O}

A presença do T. cruzi em animais silvestres da Amazônia brasileira, entre eles marsupiais, quirópteros, roedores, edentados e primatas foi relatada por Barretto (1964) e Deane (1964). Inquéritos sorológicos realizados por Coura et al. (1999) demonstraram um alto percentual de soropositividade para anticorpos anti T.cruzi. Esses autores também relataram que $20,7 \%$ das pessoas soropositivas (coletores de piaçava) reconheceram os triatomíneos como insetos presentes em seu ambiente de trabalho e destas $30 \%$ informaram terem sido picados por estes insetos. Por tratar-se de uma espécie silvestre, encontrada em uma região onde a maior preocupação não é a doença de Chagas, $R$. brethesi tem sido pouco estudada, e os conhecimentos sobre sua biologia são restritos. Estudos sobre o comportamento alimentar desta espécie podem contribuir como indicadores do potencial de transmissão do T. cruzi e auxiliar no controle dos vetores. Torna-se imprescindível dar continuidade aos estudos sobre vetores potenciais na região. Este conhecimento pode contribuir para as avaliações sobre o risco de instalação da doença de Chagas entre as populações desta área e indicar quais espécies têm maior capacidade de se adaptarem ao ambiente peri e intradomiciliar.

O primeiro trabalho realizado em laboratório sobre o ciclo biológico de $R$. brethesi foi publicado por Mascarenhas em 1990, ou seja, 71 anos depois da descrição da espécie. Neste trabalho foi avaliado o período de desenvolvimento desta espécie em temperatura ambiente que apresentou temperatura média de $26^{\circ} \mathrm{C}$ e umidade relativa de $80 \%$. Sob estas condições os autores observaram o período médio de desenvolvimento, da incubação dos ovos até o estádio adulto, em 139 dias, período este superior ao registrado no presente trabalho que foi de 115 dias.

Número de repastos realizados - Este aspecto é reconhecidamente um dos mais importantes quanto à probabilidade de infecção e ou transmissão do T. cruzi, uma vez que o contato ativo entre vetor e hospedeiro ocorre no momento da alimentação (Juarez 1970, Rocha et al. 1994, 1997). No presente trabalho $R$. brethesi apresentou uma média baixa de números de repastos por estádio, necessitando em média de apenas 1 repasto para a ocorrência de ecdises até o 50 estádio. Na fase imaginal, a média de repastos por inseto foi de 7 alimentações, chegando ao máximo de 27 repastos (Tabela II). Estes resultados indicam um excelente aproveitamento da alimentação na fase ninfal, o que parece ter dispensado a necessidade de outros repastos em um mesmo estádio de desenvolvimento. A voracidade destes insetos tem sido relatada desde a década de 1920 por Matta (1922) que informou que os piaçabeiros apresentavam inflamações na pele, causadas pela picada de $R$. brethesi e que estas ocorriam por quase todo o corpo dos indivíduos. Este contato também foi observado por Mascarenhas (1991) e Coura et al. (1993, 1994 e 1995). A capacidade de se alimentar de forma rápida e eficiente facilitaria a realização de repastos sangüíneos durante o período diurno utilizando o homem como hospedeiro. Segundo Galvão et al. (2001) o número de repastos realizados tem implicâncias epidemiológicas, já que quanto mais contatos ocorrerem entre vetor e hospedeiro mais aumentam as probabilidades de infecção ou transmissão de T. cruzi. Mas, por outro lado, quanto menos o vetor se expuser na procura pelo alimento, mais aumentam suas chances de sobrevivência. Vários autores já chamavam a atenção para a necessidade de um único repasto para que ocorressem ecdises, entre eles Brasileiro e Perondini (1974) que excluíam as ninfas de 5 estádio. Os resultados obtidos no presente trabalho demonstraram que esta espécie possui um excelente desenvolvimento quando alimentada em camundongos.

Sangue ingerido - Os aspectos biológicos, importantes do ponto de vista epidemiológico, estão relacionados à quantidade de sangue ingerida pelos triatomíneos. Esta capacidade de ingestão irá refletir na oviposição, no período de desenvolvimento, na capacidade de resistência ao jejum e na dispersão ativa através do vôo. $R$. brethesi 
apresentou um aumento progressivo na quantidade de sangue ingerida, com diminuição apenas na fase adulta, o que também é observado em várias outras espécies de triatomíneos em todos os gêneros. Em comparação com R. pictipes e R. prolixus (Buxton 1930, Rocha et al. 1994, $1997), R$. brethesi foi a que necessitou de menor quantidade de sangue em todos os estádios observados.

Padrões de defecação - O tempo que o inseto leva para defecar, após o repasto sangüíneo, vai determinar o local de realização da defecação, com conseqüente influência no processo de transmissão do T. cruzi, (Piesman \& Sherlock 1983). Existem espécies, como T. nitida (Galvão et al. 1995), que apesar de alimentarem-se com grandes quantidades de sangue não defecam rapidamente, enquanto outras o fazem. $R$. brethesi apresentou um alto percentual de defecações, durante o período de observação (até 10 min após a alimentação), o que a torna uma espécie importante quanto à sua competência vetorial.

Período de desenvolvimento ninfal - O período de desenvolvimento da eclosão até a muda imaginal, ocorreu em 115 dias em média. Pode ser considerado rápido se comparado a outras espécies do gênero, como $R$. pictipes que apresentou no mesmo período média de 238 dias (Rocha et al. 1994). Esta aceleração no período de desenvolvimento pode ter sido uma conseqüência do aproveitamento alimentar desta espécie, que por necessitar de poucos repastos para a ocorrência de ecdises, atinge de forma rápida a fase adulta. $\mathrm{O}$ período de desenvovimento ninfal de $R$. brethesi ficou próximo ao observado para $R$. neglectus por Perlowagora-Szumlewicz (1975), para $R$. neivai por Carcavallo et al. (1976) e para $R$. prolixus por Lent e Valderrama (1977).

Percentual de mortalidade - As ninfas de R. brethesi não apresentaram um padrão de mortalidade regular; os maiores percentuais foram observados no $1 \underline{0}$ e 4으 estádios, o que não é considerado por vários autores como comum nas espécies de triatomíneos que geralmente apresentam taxas elevadas de mortalidade no $1^{0} \underline{\text { e }}$ 5ㅇ estádios de desenvolvimento (Lent \& Valderrama 1977, Rocha et al. 1994, 1997, Martínez-Ibarra et al. 2003). A principal causa de mortalidade no 1 o estádio foi a dificuldade que estas ninfas apresentaram para se alimentar, chegando a morrer em jejum. Com relação ao percentual total de mortalidade $(16,3 \%)$, pode ser considerado muito baixo quando comparado com resultados obtidos para outras espécies em laboratório. Lent e Valderrama (1977) e Rocha et al. (1994) trabalhando com $R$. pictipes registraram percentual de mortalidade de 33 e $38 \%$, respectivamente. Taxas de mortalidade entre 10 e $20 \%$ já no 1 o estádio também foram observadas em espécies dos gêneros Triatoma e Panstrongylus (Corrêa 1962, Rabinovich 1972, Feliciangeli \& Rabinovich 1985).

Os resultados obtidos no presente trabalho mostram que $R$. brethesi apresenta um bom aproveitamento do repasto sanguiíneo e um rápido desenvolvimento ninfal em laboratório, o que parece refletir, segundo estudos realizados em campo por outros autores, o comportamento desta espécie no ambiente natural.

\section{AGRADECIMENTOS}

Ao Dr. Tobby Barret do Instituto de Pesquisas da Amazônia, pelos espécimes de Rhodnius brethesi que deram origem à colônia.

\section{REFERÊNCIAS}

Barreto MP 1964. Reservatórios do Trypanosoma cruzi nas Américas. Rev Bras Malar 16: 527.

Brasileiro VLF, Perondini ALP 1974. Biologia do T. brasiliensis (Hemiptera, Reduviidae,Triatominae). I. Tempo de sucção e repleção de ninfas de 4으 e 5을 estádios. Rev Bras Ent 18: 43-50.

Buxton PA 1930. The biology of a blood sucking bug Rhodnius prolixus. Trans Entomol Soc London 78: 227-236.

Carcavallo RU, Curto de Casas SI, Sherlock IA, Galíndez-Girón I, Jurberg J, Galvão C, Mena Segura CA 1999. Geographical distribution and altilatitudinal dispersion. In R Carcavallo, I Galíndez-Girón, J Jurberg, H Lent (orgs.), Atlas of Chagas Disease Vectors in the Americas, Vol. III, Editora Fiocruz, Rio de Janeiro, p. 747-792.

Carcavallo RU, Tonn RJ, Jimenez JC 1976. Notas sobre la biologia, ecologia y distribución geográfica de Rhodnius neivai Lent, 1953 (Hemiptera, Reduviidae). Bol Dir Malariol y San Amb 16: 169-171.

Correa FMA 1962. Estudo compartivo do ciclo evolutivo do Triatoma infestans alimentado em diferentes animais (He-miptera, Reduviidae). Papéis Avulsos do Dept Zool Sec Agric SP 15: 177-200.

Coura JR, Arboleda MN, Willcox HPF 1993. Doença de Chagas na Amazônia brasileira. Rev Soc Bras Med Trop 26 (Supl. 2): $15-17$.

Coura JR, Arboleda MN, Willcox HPF 1995. Chagas disease in the Brazilian Amazon. II - A serological survey. Rev Med Trop São Paulo 37: 103-107. Rev Soc Bras Med Trop 27: 251-253.

Coura JR, Barrett TV, Arboleda MN 1994. Ataque de populações humanas por triatomíneos silvestres no Amazonas: uma nova forma de transmissão da infecção chagásica? Rev Soc Bras Med Trpo 27: 251-253.

Coura JR, Junqueira ACV, Boia MN, Fernandes O 1999. Chagas disease: from bush to huts and houses. Is it the case of the Brazilian Amazon? Mem Inst Oswaldo Cruz 94(Suppl.1): 379-384.

Coura JR, Junqueira AC, Fernandes O, Valente AS, Miles MA 2002. Emerging Chagas disease in Amazonian Brazil. Trends in Parasitol 18: 171-176.

Deane LM1964. Animal reservoirs of Trypanosoma cruzi in Brazil. Rev Brasil Malariol D Trop 16: 27-48.

D’ Allesandro A, Barreto P, Duarte CA 1971. Distribution of triatominae transmited trypanosomiase in Colombia and new records of the bugs and infections. J Med Entomol 8: 159-172.

Dias JCP, Prata A, Schofield JC 2002. Doença de Chagas na Amazônia: esboço da situação atual e perspectiva de prevenção. Rev Soc Bras de Med Trop 35: 669-678.

Feliciangeli MD, Rabinovich J 1985. Vital statistics of Triatominae (Hemiptera:Reduviidae) under laboratory conditions. II. Triatoma maculata. J Med Entomol 22: 4348.

Galvão C, Carcavallo RU, Rocha DS, Jurberg J 2003. A checklist of the current valid species of the subfamily Triatominae Jeannel, 1919 (Hemiptera, Reduviidae) and their geographical distribution, with nomenclatural and taxonomic notes. Zootaxa 202: 1-36.

Galvão C, Jurberg J, Cunha V, Mello RP 1995. Biologia do Triatoma nitida Usinger, 1939 em laboratório (Hemiptera, Reduviidae). Mem Inst Oswaldo Cruz 90: 657-663. 
Galvão C, Rocha DS, Cunha V, Jurberg J, Carcavallo RU 2001. Tempo de alimentação e defecação das ninfas de Triatoma melanosoma Martínez, Olmedo \& Carcavallo, 1987 (Hemiptera, Reduviidae) em diferentes condições de temperatura e umidade. Revta Bras Zool 18: 233-242.

Juarez E 1970. Comportamento do Triatoma infestans sob várias condições de laboratório. Rev Saúde Públ São Paulo 4: 147166.

Lent H, Valderrama A 1977. Observações em laboratório, sobre o ciclo evolutivo de Rhodnius prolixus Stal,1859, R. pictipes Stal,1872 e R. neivai Lent, 1953. Rev Brasil Biol 37: 325344.

Martínez-Ibarra JÁ, López MN, Robles MRH, Guillén YG 2003. Influence of the blood meal source on the biology of Meccus picturatus Usinger 1939 (Hemiptera: Reduviidae: Triatominae) under laboratory conditions. Mem Inst Oswaldo Cruz 98: 227-232.

Mascarenhas BM 1990. Triatomíneos da Amazônia. Sobre o ciclo evolutivo de Rhodnius brethesi Matta, 1919 (Hemiptera, Reduviidae: Triatominae). Bol Mus Par Emilio Goeldi Série Zol 6: 191-202.

Mascarenhas BM 1991. Triatomíneos da Amazônia: sobre o habitat e algumas considerações comportamentais de Rhodnius brethesi Matta, 1919 (Hemiptera, Reduviidae: Triatominae) na região do médio Rio Negro, Amazonas. Bol Mus Par Emilio Goeldi Série Zol 7: 107-116.

Matta A 1922. Sobre o gênero Rhodnius do Amazonas.
Amazonas Med 5: 161-162.

Perlowagora-Szumlewicz A 1975. Laboratory colonies of Triatominae, biology and population dinamics. In American Tripanosomiasis Research. PAHO Scient Publ 318: 6382.

Piesman J, Sherlock IA 1983. Factors controlling the volume produced by triatominae vectors of Chagas disease. Acta Trop 40: 351-358.

Rabinovich JE 1972. Vital statistics of Triatominae (Hemiptera: Reduviidae) under laboratory conditions. I. Triatoma infestans Klug. J Med Ent 9: 351-370.

Rebelo JMM, Barros VLL, Mendes WA 1998. Espécies de Triatominae (Hemiptera: Reduviidae) do Estado do Maranhão, Brasil. Cad Saúde Públ 14: 187-192.

Rocha DS, Fonseca AH, Costa FA, Jurberg J, Galvão C. 1997. Desenvolvimento de Rhodnius pictipes Stal, 1872 alimentado através de membrana de silicone e em camundongos (Hemiptera, Reduviidae, Triatominae). Mem Inst Oswaldo Cruz 92: 553-558.

Rocha DS, Galvão C, Jurberg J 1994. Biologia do Rhodnius pictipes Stal, 1872 em condições de laboratório (Hemiptera, Reduviidae, Triatominae). Mem Inst Oswaldo Cruz 89: 265270.

Schofield CJ 1985. Population dynamics and control of Triatoma infestans. Ann Soc Belge Med Trop 65: 149-164.

Valente SAS, Valente VC 1993. Situação da doença de Chagas na Amazônia. Rev Soc Bras Med Trop 26(Supl. 29): 68-70. 